\title{
Progressive CT system technology and experience improve the perceived preoperative stage of gastric cancer
}

\author{
Guy R.J.C. Blackshaw ${ }^{1}$, Michael R. Stephens ${ }^{1}$, Wyn G. Lewis ${ }^{1}$, Jamie Boyce ${ }^{1}$, John D. Barry ${ }^{1}$, \\ Paul Edwards ${ }^{1}$, Miles C. Allison ${ }^{2}$, and Gerald V. Thomas ${ }^{3}$ \\ ${ }^{1}$ Department of Surgery, Royal Gwent Hospital, Cardiff Road, Newport, NP20 2UB, UK \\ ${ }^{2}$ Department of Gastroenterology, Royal Gwent Hospital, Newport, UK \\ ${ }^{3}$ Department of Radiology, Royal Gwent Hospital, Newport, UK
}

\begin{abstract}
Background. Our goals were to measure the accuracy of specialist helical computed tomography (CT) in the preoperative staging of gastric cancer, to determine the relative benefit of progressive CT system technology, and to determine the magnitude of any learning curve in radiological interpretation. Methods. One hundred patients (median age, 70 years; range 27-86 years; 68 male) underwent a preoperative CT ( 73 helical [hCT], 27 multislice [mCT]), performed by a single specialist radiologist, followed by surgery within 3 weeks. The strength of the agreement between the perceived CT stage and the histopathological stage was determined for each CT system and also for four serial cohorts of 25 patients, by the weighted Kappa statistic (Kw).

Results. The Kw values for $\mathrm{T}, \mathrm{N}, \mathrm{M1}$ liver, and $\mathrm{M} 1$ peritoneal stage were $0.40,0.18,0.36$, and 0.09 for $\mathrm{hCT}$, compared with 0.57, 0.67, 0.66 (all $P<0.001)$, and $0.24(P=0.06)$ for $\mathrm{mCT}$. Serial $\mathrm{Kw}$ for $\mathrm{T}$ and $\mathrm{N}$ stages improved from 0.26 and -0.14 in the first quartile of patients to 0.61 and $0.73(P<0.001)$ in the last quartile of patients.

Conclusion. The role of $\mathrm{CT}$ in the preoperative staging of gastric cancer is becoming stronger as CT technology improves.
\end{abstract}

Key words Gastric cancer · Surgery · Computed tomography

\section{Introduction}

Gastric cancer is now the second commonest cancer worldwide, and the most recent United Kingdom data estimated that there are 10000 new diagnoses and 7500 deaths from gastric cancer each year [1,2]. Surgery re-

Offprint requests to: W.G. Lewis

An original article presented in part at the Fifth International Gastric Cancer Congress, Rome, May 2003; Eleventh UEGW, Madrid, November 2003; and Digestive Disease Week, New Orleans, May 2004.

Received: August 23, 2004 / Accepted: November 1, 2004 mains the prime therapeutic modality for gastric cancer, but over $80 \%$ of patients are over the age of 65 years at diagnosis, comorbidity is common, and as many as one in three patients have incurable disease at presentation [3]. In the recently published audit of the treatment of esophagogastric cancer in Wales, many surgeons undertook small caseloads, staging strategies varied widely, and open-and-close operations were common [4]. Stage-directed management, tailored to individual patients, is clearly desirable if the benefits of greater subspecialization and a multidisciplinary approach are to be realized [5]. Computed tomography (CT), endogastric ultrasound, and laparoscopy all have a role to play in the preoperative staging strategy of gastric cancer [6]. Although CT represents the most widely used imaging technique for the preoperative staging of patients with gastric cancer, the modality has limitations in detecting local invasion of tumors and lymph node metastases [710]. Moreover, we have shown previously that discrepancies exist, even among experienced radiologists, in the interpretation of subtle radiological signs, and that special interest radiology improves the perceived preoperative stage of gastric cancer [11].

The aim of this study was to measure the accuracy of serial special interest CT over time and when enhanced by regular multidisciplinary team feedback, and to determine the relative benefit of progressive CT system technology and the magnitude of any learning curve in radiological interpretation.

\section{Patients and methods}

Between July 1, 1997, and April 30, 2004, our center managed 267 patients with gastric cancer. Of these, 100 patients underwent both preoperative $\mathrm{CT}$ and operative assessment. Each patient had a preoperative histological diagnosis of gastric adenocarcinoma established by gastroscopy and biopsy. The median age was 70 years 
(range, 27 to 86 years). Sixty-eight patients were male and 32 were female. The CT examinations undertaken before April 10, 2002, were performed using a helical Siemens Somatom +4 CT system (Siemens, Munich, Germany; 73 patients); and after April 10, 2002, the CT examinations were performed using a multislice Toshiba Aquilon volume acquisition spiral CT system (Toshiba, Tokyo, Japan; 27 patients). All patients underwent a contrast enhanced CT within 3 weeks of operation. Thirty minutes before scanning, after an overnight fast, the first 33 patients were asked to drink $250 \mathrm{ml}$ of Microcat (Guernet, Roissy DdCr Cedex, France) to opacify the small bowel. This policy was changed in June 1999, when use of oral contrast was discontinued. Instead, all patients were asked to drink as much of a 1000-ml water load as they could tolerate. Intravenous contrast Niopam $300(100 \mathrm{ml}$; Merck Pharmaceuticals, Middlesex, UK) was delivered by power injector, at a rate of $3 \mathrm{ml}$ per $\mathrm{s}$, through a cannula in the antecubital fossa. None of the patients received antimotility medication. The exact CT scanning protocol varied according to the specific $\mathrm{CT}$ system used.

\section{Helical CT system (Siemens Somatom +4)}

All patients were scanned in the supine position. Initially, a volume acquisition of the thorax was performed, using 10-mm slice thicknesses, a reconstruction index of $8 \mathrm{~mm}$, and a table speed of $10 \mathrm{~mm}$ per s. A second volume acquisition of the abdomen from the dome of the diaphragm to the pelvic brim was performed, using 10-mm slice thicknesses with a reconstruction index of $5 \mathrm{~mm}$. This meant that slices were acquired every $10 \mathrm{~mm}$, but reconstructed at 5-mm intervals, which produced overlapping slices. The second volume was timed to begin liver imaging $60-70 \mathrm{~s}$ after the onset of contrast injection.

\section{Volume acquisition multislice CT system (Toshiba Aquilon)}

This CT system used 1-mm slices taken $1 \mathrm{~mm}$ apart, giving a reconstruction index of $1 \mathrm{~mm}$. The pitch of the system (ratio of table movement: rotation of the tube) was set at 1.438 . For the purposes of this study, adjacent organs were considered to be involved if there was CT evidence of direct spread of the tumor into adjacent organs. Specific areas of fat plane effacement were also compared with adjacent evidence of fat plane preservation. Lymph nodes were considered to be involved if they were greater than $1 \mathrm{~cm}$ in diameter [12-14]. The results of the single Consultant Radiologist (G.V.T.), who worked as part of the upper gastrointestinal multidisciplinary team, were divided into chronological groups of 25 patient quartiles (Table 1). Patients who
Table 1. Details of the patients related to position in the series

\begin{tabular}{lcccc}
\hline & \multicolumn{4}{c}{ Series number } \\
\cline { 2 - 5 } & $1-25$ & $26-50$ & $51-75$ & $76-100$ \\
\hline Median age (years) & 74 & 65 & 68 & 68 \\
Sex m:f & $17: 8$ & $17: 8$ & $16: 9$ & $18: 7$ \\
Stage of disease & & & & \\
I & 2 & 2 & 4 & 4 \\
II & 3 & 4 & 2 & 5 \\
IIIA & 4 & 5 & 3 & 3 \\
IIIB & 7 & 1 & 6 & 1 \\
IV & 9 & 13 & 10 & 12 \\
\hline
\end{tabular}

Figures are numbers of patients

were perceived to have advanced stage disease (T3/4, $\mathrm{N} 1 / 2$, and equivocal M1) underwent a preoperative staging laparoscopy $(n=81)$. No patients received preoperative neoadjuvant chemotherapy. Forty-four patients underwent a curative radical D2 gastrectomy, 12 underwent a palliative resection, and the remaining 44 patients underwent laparoscopy only $(n=29)$, palliative bypasses $(n=7)$, or laparotomy only $(n=8)$. All patients underwent operative assessment as suggested by Rohde et al. [15].

\section{Statistical analysis}

The findings from CT and intraoperative surgical assessments were compared with the results of postoperative histopathological assessment. Agreement between the perceived preoperative stage of gastric cancer, as determined by $\mathrm{CT}$, and the postoperative histopathological assessment was determined using the weighted Kappa statistic (Kw) [16]. The value of Kappa has a maximum of 1.00 when agreement is perfect, a value of zero indicates agreement no better than chance, and negative values show worse than chance agreement. We tested the hypothesis $\mathrm{Kw}=0$ and assessed the value of $\mathrm{Kw}$ for strength of agreement with the guidelines of Landis and Koch [17]. The sensitivity and specificity, positive predictive value (PPV), and negative predictive value (NPV) were also estimated. Categorical data were further analyzed by means of the $\chi^{2}$ distribution [16]. Data analysis was carried out with the Statistical Package for Social Sciences (SPSS) version 11.5 (SPSS, Chicago, IL, USA).

\section{Results}

\section{Relative accuracy of CT stage related to $C T$ system} (Table 2)

The relative accuracy of the perceived preoperative $\mathrm{T}$, $\mathrm{N}$, and $\mathrm{M}$ stages with respect to the CT system is demon- 
Table 2. Relative accuracy of CT system technology in the preoperative staging of gastric cancer

\begin{tabular}{|c|c|c|c|c|c|c|}
\hline \multirow{3}{*}{ Number of patients } & \multicolumn{3}{|c|}{$\mathrm{hCT}$} & \multicolumn{3}{|c|}{$\mathrm{mCT}$} \\
\hline & \multicolumn{3}{|c|}{72} & \multicolumn{3}{|c|}{28} \\
\hline & Sens & Spec & $\mathrm{Kw}$ & Sens & Spec & $\mathrm{Kw}$ \\
\hline $\mathrm{T}$ & $63 \%$ & $83 \%$ & $0.40 * *$ & $69 \%$ & $87 \%$ & $0.57 * *$ \\
\hline $\mathrm{N}$ & $63 \%$ & $39 \%$ & 0.18 & $89 \%$ & $78 \%$ & $0.67 * *$ \\
\hline M1L & $45 \%$ & $90 \%$ & $0.36^{*}$ & $80 \%$ & $91 \%$ & $0.66^{*}$ \\
\hline M1P & $6 \%$ & $100 \%$ & 0.09 & $25 \%$ & $100 \%$ & 0.24 \\
\hline
\end{tabular}

$* P<0.05 ; * * P<0.001$

$\mathrm{M} 1 \mathrm{~L}$, liver metastases; M1P, peritoneal metastases

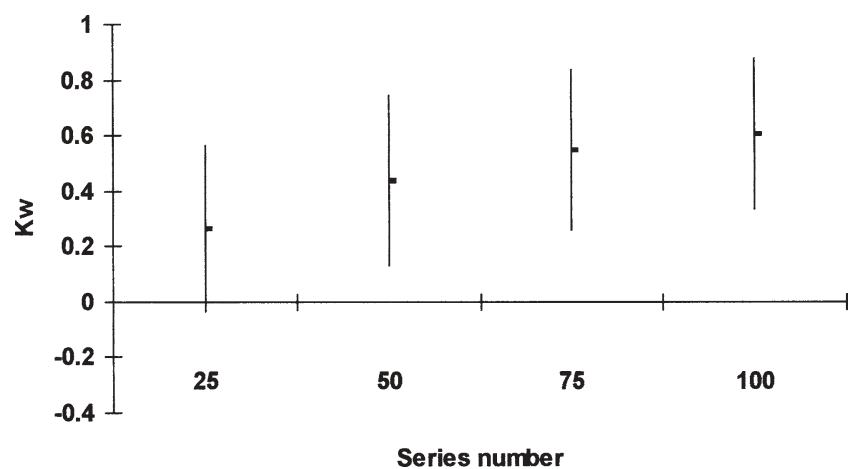

Fig. 1. Strength of agreement $(K w)$ and $95 \%$ confidence interval (CI) between computed tomography (CT) $\mathrm{T}$ stage and histopathological $\mathrm{T}$ stage related to experience of radiologist

strated in Table 2. The strength of agreement between the overall $\mathrm{CT}$ stage and the histopathological stage was 0.28 for hCT (95\% confidence interval [CI], 0.14$0.41 ; P<0.001)$ compared with 0.53 (95\% CI, $0.30-0.76$; $P<0.001)$ for $\mathrm{mCT}$.

Relative accuracy of $C T T$ and $N$ stage related to $C T$ system in stage II or III gastric cancer

A total of 44 patients had stage II or III cancer (14, stage II; 30, stage III). The strengths of agreement between the CT T stage and the histopathological $\mathrm{T}$ stage were 0.30 (95\% CI, 0.03-0.56; $P=0.021$ ) for hCT compared with $0.58(95 \% \mathrm{CI}, 0.091-1.00 ; P=0.007)$ for $\mathrm{mCT}$. The strengths of agreement between the CT N stage and the histopathological N stage were -0.197 (95\% CI -0.521 to $0.127 ; P=0.236)$ for hCT compared with $0.40(95 \%$ CI -0.168 to $0.968 ; P=0.197)$ for $\mathrm{mCT}$.

\section{Relative accuracy of CT T stage related to experience of radiologist (Fig. 1)}

The strengths of agreement between the CT T stage and the histopathological $\mathrm{T}$ stage improved from fair and

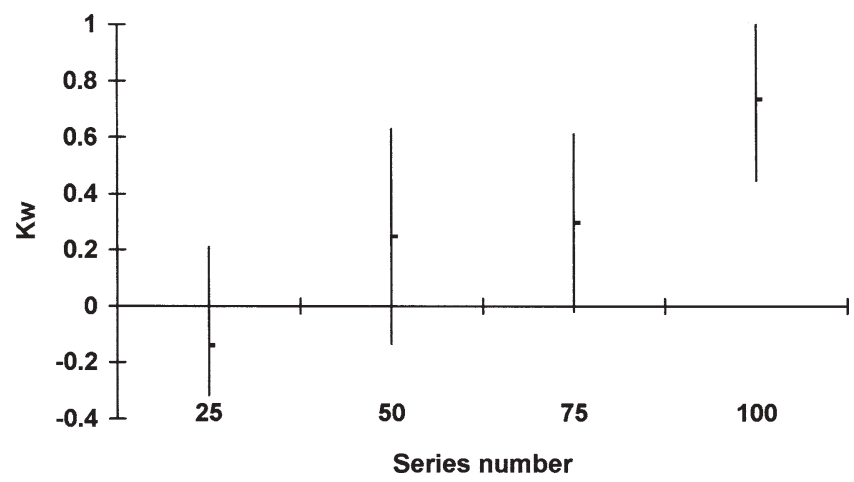

Fig. 2. Strength of agreement $(K w)$ and $95 \%$ CI between CT $\mathrm{N}$ stage and histopathological $\mathrm{N}$ stage related to experience of radiologist

moderate (Kw, 0.26; $P=0.066 ; \mathrm{Kw}, 0.44 ; P=0.004$; $\mathrm{Kw}, 0.55 ; P<0.001)$ in the first 75 patients to good $(\mathrm{Kw}$, $0.61 ; P<0.001)$ in the latter 25 patients.

Relative accuracy of CT $N$ stage related to experience of radiologist (Fig. 2)

The strengths of agreement between the CT N stage and the histopathological $\mathrm{N}$ stage remained poor or fair for the first 75 patients $(\mathrm{Kw},-0.14 ; P=0.48 ; \mathrm{Kw}, 0.25$; $P=0.2 ; \mathrm{Kw}, 0.30 ; P=0.078)$, but improved in the latter 25 patients to good $(\mathrm{Kw}, 0.73 ; P<0.001)$.

Relative accuracy of CT M1 liver and peritoneum stages related to experience of radiologist

(Figs. 3 and 4)

The strengths of agreement between the CT M1 liver stage and the histopathological M1 liver stage ranged from fair to good throughout $(\mathrm{Kw}, 0.62 ; P=0.002 ; \mathrm{Kw}$, $0.19 ; P=0.35 ; \mathrm{Kw}, 0.41 ; P=0.04$ and $\mathrm{Kw}, 0.63 ; P=$ 0.001 ), and for M1 peritoneum stage ranged from poor to fair throughout $(\mathrm{Kw}, 0.19 ; P=0.10 ; \mathrm{Kw}, 0.19 ; P=$ $0.10 ; \mathrm{Kw}, 0.16 ; P=0.14$ and $\mathrm{Kw}, 0.47 ; P=0.006$ ). 


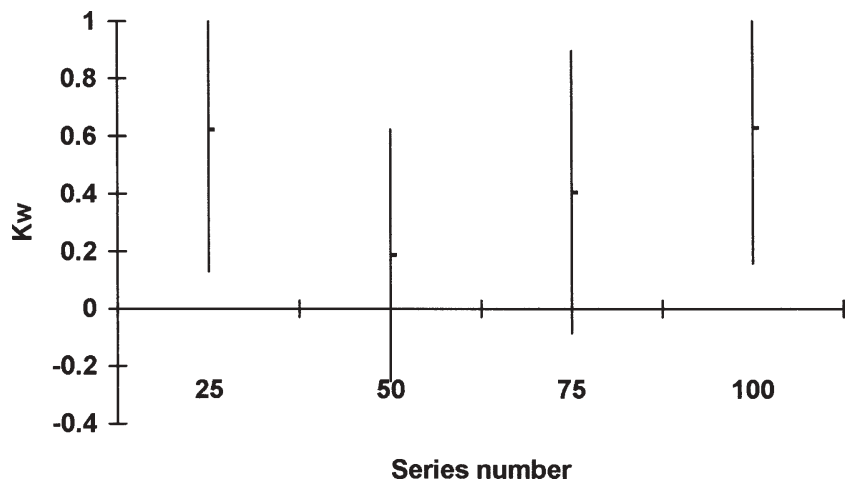

Fig. 3. Strength of agreement $(K w)$ and $95 \%$ CI between CT M1 liver stage and histopathological M1 liver stage related to experience of radiologist

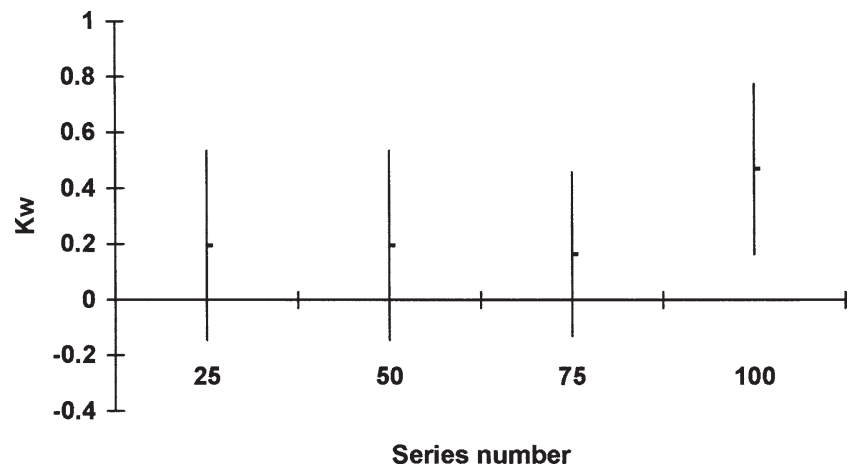

Fig. 4. Strength of agreement $(K w)$ and $95 \%$ CI between CT M1 peritoneal stage and histopathological M1 peritoneal stage related to experience of radiologist

\section{Relative accuracy of overall CT stage related to experience of radiologist}

The strengths of agreement between the overall CT stage and the overall histopathological stage gradually improved from fair to good (Kw, 0.24; $P=0.01$; $\mathrm{Kw}, 0.22 ; P=0.051 ; \mathrm{Kw}, 0.37 ; P<0.001$, and $\mathrm{Kw}, 0.72$; $P<0.001)$.

\section{Relative accuracy of CT T stage related to site of tumor}

The staging of tumors of the gastric cardia was more accurate when compared to more distal gastric tumors, with respect to $\mathrm{T}$ stage (cardia $\mathrm{T}$ stage $\mathrm{Kw}, 0.73$; $P<0.001$; gastric body Kw, $0.56 ; P=0.001$; and antrum Kw, 0.31; $P=0.025)$.

\section{Details of the surgical procedures performed}

Undetected distant liver or peritoneal metastases were discovered in 21 of the 100 patients (13 peritoneum alone, 7 liver alone, and 1 liver and peritoneum). These metastases were detected by laparoscopy in 12 patients and by laparotomy in 4 patients (open-and-close); 3 patients underwent palliative bypass and 2 patients underwent palliative resection. Five of the first quartile of 25 patients had undetected distant metastases, and this proportion was 8 in the second quartile, 4 in the third quartile, and 3 in the fourth quartile of patients.

\section{Discussion}

Accurate stage-directed management of gastric cancer tailored to individual patients is essential in order to facilitate well-informed and appropriate treatment, if the benefits of greater medical subspecialization and a multidisciplinary team approach are to be realized. The principal findings of this study were that special interest radiology, within the framework of regular multidisciplinary team meetings, allied to progressive developments in CT system technology, improved the accuracy of the perceived preoperative stage of gastric cancer over time. The detection of local tumor invasion improved over twofold, while the detection of suspicious metastases to lymph nodes improved sevenfold. The detection of distant metastases, either liver or peritoneal, was satisfactory throughout the series.

As far as we are aware, only two studies have assessed the statistical strengths of agreement between CT and the histopathological stage of gastric cancer. The original study by Ziegler et al. [18] compared CT, endosonography and operative findings with the final TN histopathological stage of gastric cancer, while the second study, conducted by our group, reported the influence of special interest radiology on the accuracy of $\mathrm{CT}$ in this regard [11]. The strengths of agreement $(\mathrm{Kw})$ reported for $\mathrm{T}$ and $\mathrm{N}$ stages by $\mathrm{CT}$ were 0.209 and 0.071 by Ziegler et al. [18] compared with 0.314 and 0.350 by Barry et al. [11]. The relative accuracy of our specialist radiologist in this study improved twofold for both $\mathrm{T}$ and $\mathrm{N}$ stage after 75 cases had been discussed at the multidisciplinary team forum. Endogastric ultrasound (EUS) has been championed as the answer to some of the limitations of CT [18], but in reality the two modalities are complementary. Certainly, EUS is the only modality able to assess accurately the $\mathrm{T}$ stage of a gastric cancer. Pollack et al. [19] in a review, described the accuracy of endoluminal ultrasound in staging gastric cancer as $77 \%$ for $\mathrm{T}$ stage and $69 \%$ for $\mathrm{N}$ stage. The strength of agreement between EUS and histopathological T and $\mathrm{N}$ stages reported by Ziegler et al. [18] was certainly far more impressive than those achieved by CT alone with a Kw of 0.873 (very good) for T stage and 0.597 (moderate) for $\mathrm{N}$ stages. Our own multidisciplinary team (MDT) enhanced CT staging results clearly do not match this level of agreement for $\mathrm{T}$ stages, but 
compare favorably with the strength of agreement reported for $\mathrm{N}$ stages, with good agreement after 100 cases were discussed allied to the latest generation of multi-slice helical CT systems. Concerns have been raised regarding the accuracy of $\mathrm{CT}$ when assessing junctional tumors of the upper third of the stomach [20], where it is possible that scanning obliquely may result in misinterpretation of the depth of tumor penetration [20]. This concern did not emerge as a practical problem in the present study, with the strength of agreement between the CT T stage for tumors of the cardia twofold more accurate in general than tumors of the gastric body and antrum.

Spiral contrast enhanced CT with thin collimation is described as the optimal technique in the recently published guidelines for the management of esophageal and gastric cancer [6]. Progressive improvements in CT system technology, based upon power slip ring technology, enabling faster, artifact-free scanning, clearly influenced the strengths of agreement between the perceived preoperative CT stages and the histopathological TNM stages in all categories in this study. Moreover, the accuracy of the CT T stage, as determined by the multislice helical CT system, approached the accuracy of the EUS T stage described by Ziegler et al. [18], and, with regard to the CT $\mathrm{N}$ stage, bettered the accuracy of EUS $\mathrm{N}$ Stage by $23 \%$. This was of particular benefit in the assessment of patients with stage II and III tumors, where the strength of agreement between $\mathrm{MCT}$ and the final histopathological $\mathrm{T}$ and $\mathrm{N}$ stages was moderate and fair, compared with fair and poor for hCT. Patients with stage III cancers may potentially benefit from neoadjuvant therapy, and an accurate perceived preoperative stage would facilitate multidisciplinary decisionmaking in this regard. The most valuable information for the practice of radical D2 gastric resections is the presence or otherwise of distant metastases (M1), which determines whether or not a curative $\mathrm{R} 0$ resection can be performed. Liver metastases are reported to be relatively uncommon at presentation in patients with gastric cancer $(8 \%-10 \%)$, although as many as one in two metastases are missed on CT alone [20]. In contrast to the above data, as many as $43 \%$ of patients in our practice have distant metastases at presentation - $34 \%$ in this study alone [21]. Liver metastases were detected with a relatively uniform accuracy throughout the period of this study. Indeed, the overall $\mathrm{Kw}$ for M1 liver when all stage IV cases are taken into consideration would be 0.77 (95\% CI, 0.68-0.86; $P=0.0001)$.

In conclusion, a mixed learning curve has emerged from the results of this study, reflecting the relative accuracy of $\mathrm{CT}$ in assessing the $\mathrm{T}, \mathrm{N}$, and $\mathrm{M}$ stages of gastric cancer. The Guidance on Commissioning Cancer Services, published by the NHS Executive [22], highlighted surgical subspecialization, and the develop- ment of a multidisciplinary team approach, as key factors in the successful management of patients with upper gastrointestinal malignancies. Patient selection is clearly important if any survival advantage for resection is to be realized, and the findings of this study underscore the impact of high-volume experience, allied to surgical and histopathological feedback, in refining preoperative diagnosis and stage. Furthermore, with progressive improvements in CT system technology, the role of $\mathrm{CT}$ in the preoperative staging of gastric cancer is becoming stronger.

Acknowledgments G.R.J.C. Blackshaw and J.D. Barry were supported in part by a grant from the ASTRA foundation.

\section{References}

1. CRC (Cancer Research Campaign). Factsheet 1.1 Incidence-UK. London: Cancer Research Campaign; 1998.

2. CRC (Cancer Research Campaign). Cancer Stats Mortality-UK. London: Cancer Research Campaign; 1999.

3. Allum WH, Powell DJ, McConkey CC, Fielding JWL. Gastric cancer: a 25-year review. Br J Surg 1989;76:535-40.

4. Pye JK, Crumplin MKH, Foster ME, Biffin A, Charles J. Oneyear survey of carcinoma of the oesophagus and stomach in Wales. Br J Surg 2001;88:278-85.

5. Cunningham D, Allum W, Weeden S. Perioperative chemotherapy in operable gastric cancer and lower oesophageal cancer: a randomized trial of the UK NCRI Upper GI Clinical Studies Group (the MAGIC trial, ISRCTN 93793971). Eur J Cancer 2003; Suppl 1(5):S18.

6. Allum WH, Griffin SM, Watson A, Colin-Jones D. Guidelines for the management of oesophageal and gastric cancer. Gut 2002;50 (Suppl V):1-23.

7. Fraser I, Nash R, James DC. Computed tomography in gastric cancer. Br J Surg 1985;72:249-50.

8. Cook AO, Levine BA, Sirinek KR, Gaskill HV. Evaluation of gastric adenocarcinoma: abdominal computed tomography does not replace celiotomy. Arch Surg 1986;121:603-6.

9. Sussman SK, Halvorsen RA, Illescas FF, Cohan RH, Saeed M, Silverman WM, et al. Gastric adenocarcinoma: CT versus surgical staging. Radiology 1988;167:335-40.

10. Andaaker L, Morales O, Hojer H, Backstrand B, Borch K, Larsson J. Evaluation of pre-operative computed tomography in gastric malignancy. Surgery 1991;109:132-5.

11. Barry JD, Edwards P, Lewis WG, Dhariwal D, Thomas GV. Special interest radiology improves the perceived preoperative stage of gastric cancer. Clin Rad 2002;57:984-8.

12. Vallgren S, Hedenbro J, Gotberg S, Walther B. Preoperative computed tomography for evaluation of tumour growth in patients with gastric cancer. Acta Chir Scand 1985;151:571-3.

13. Botet J, Lightdale CJ, Zauber AG, Gerdes H, Winawer SJ, Urmacher C, et al. Preoperative staging of gastric cancer: comparison of endoscopic US and dynamic CT. Radiology 1991;181: 426-32.

14. Maruyama M, Baba M. Gastric carcinoma: radiology of the upper gastrointestinal tract. Radiol Clin North Am 1994;32:123352.

15. Rohde H, Rau E, Koster R, Gebbensleben B, Stutzer H. A surgeon's checklist for a standardized staging procedure in patients with cancer of the stomach. Scand J Gastroenterol 1987;22 (Suppl 133):36-9. 
16. Altman DG. Practical statistics for medical research. London: Chapman \& Hall/CRC; 1991.

17. Landis JR, Koch GG. The measurement of observer agreement for categorical data. Biometrics 1977;33:159-74.

18. Ziegler K, Sanft C, Zimmer M, Felsenberg D, Stein H, Germer C, et al. Comparison of computed tomography, endosonography, and intraoperative assessment in TN staging of gastric carcinoma. Gut 1993;34:604-10.

19. Pollack BJ, Chak A, Sivak MV. Endoscopic ultrasonography. Semin Oncol 1996;23:336-46.
20. Martin IG. Staging of esophageal and gastric carcinoma. In: Daly JM, Hennessy TPJ, Reynolds JV, editors. Management of upper gastrointestinal cancer. London: WB Saunders; 1999.

21. Lewis WG, Edwards P, Barry JD, Dhariwal D, Khan S, Hodzovic I, et al. D2 or not D2? The gastrectomy question. Gastric Cancer 2002;5:29-34.

22. National Cancer Guidance Steering Group. Guidance on Commissioning Cancer Services: Improving Outcomes in Upper Gastro-Intestinal Cancer. London: NHS Executive, Department of Health; 2001. 